\title{
Management of vesicouretral using immunohistochemistry
}

\author{
Pedro Ferraz-Gameiro ; Helga Fritsch ${ }^{* * *}$; Josef Oswald ${ }^{* * *}$ \\ *Faculdade de Medicina da Universidade de Coimbra (FMUC), alfa_guest@hotmail.com \\ ${ }^{* *}$ Department for Paediatric Urology Medizinuniversität Innsbruck \\ ${ }^{* * *}$ Institute of Anatomy, Histology and Embryology, Medical University of Innsbruck
}

The vesicouretral reflux is an important pathology that has an incidence of about $1 \%$ in children, and may be associated with renal scarring and hypertension due to kidney failure. ${ }^{1}$ The reason for this insufficient ureterovesical valve is based on congenital dysplasia, atrophy and architectural derangement of smooth muscle fibers of the distal ureteral muscle wall. The ureterovesical junction is a way of avoiding the reflux from the bladder to the higher urinary system (ureters and kidneys). This junction has a valve-like action. One important factor on the prevention of the reflux is the length of the intravesical ureter. These conditions (ureterovesical junction and length of the intravesical ureter) are, respectively, an active and a passive way of preventing reflux ${ }^{1}$.

This study gives particular emphasis to the fact that the reflux may be associated with dysplasia of the muscle cells that constitute the distal portion of the ureter, possibly resulting in a weaker active sphincter. The samples that were used for the study were obtained from ureterorenal units in children undergoing reflux surgery. The tissue preparation for light microscopy was made according to classical procedures. In the immunohistochemical analysis peroxidase labelled antibodies were used and then revealed with diaminobenzydine. Direct and indirect immunohistochemistry methods were used.

The research data was statistically evaluated with the non-parametric statistic study MannWhitney test, after proving that the values were not distributed according to the Gauss curve ( standard values) by the Shapiro-Wilk test. Each staining was compared in the study group to the results of the control group.

As one can conclude, there are several changes in the meaning not only of the extracellular matrix (ECM) but also in the number of the muscular cells in the region of the trigone of the bladder. There is a fewer number of smooth muscle cells in the distal portion of the ureter; by looking at Fig. $2 \mathrm{~A}$ and B, we can notice that there are much more smooth muscle cells in the control case than in the study case. One can deduce from this fact that smoothelin is a marker of this kind of cells. There are changes at the level of the muscle sheaths, according to changes in the ECM, as the results of the staining with antibodies against FGF2 and Collagen I prove. There was no statistical difference in the counting of the cells stained with anti-myofibroblast antibodies.

There is an underexpression of collagen I in the study cases comparing to the control cases, which may mean, that some weakness in this region of the ureter might be found by being not so strong against traction strengths, as it is typical of tissues with more collagen. As one can conclude, there are not only alterations in the number and constitution of the smooth muscle cells - dysplasia, but also changes at the level of the muscle cell sheath- changes of the ECM.

References:

[1] Oswald,J.,Brenner, E., Schwentner, C., Deibl, M., Bartsch, G., Fritsch, H. et al: The intravesical ureter in children with vesicoureteral reflux: a morphological and immunohistochemical characterization. J Urol, 170: 2423, 2003. 


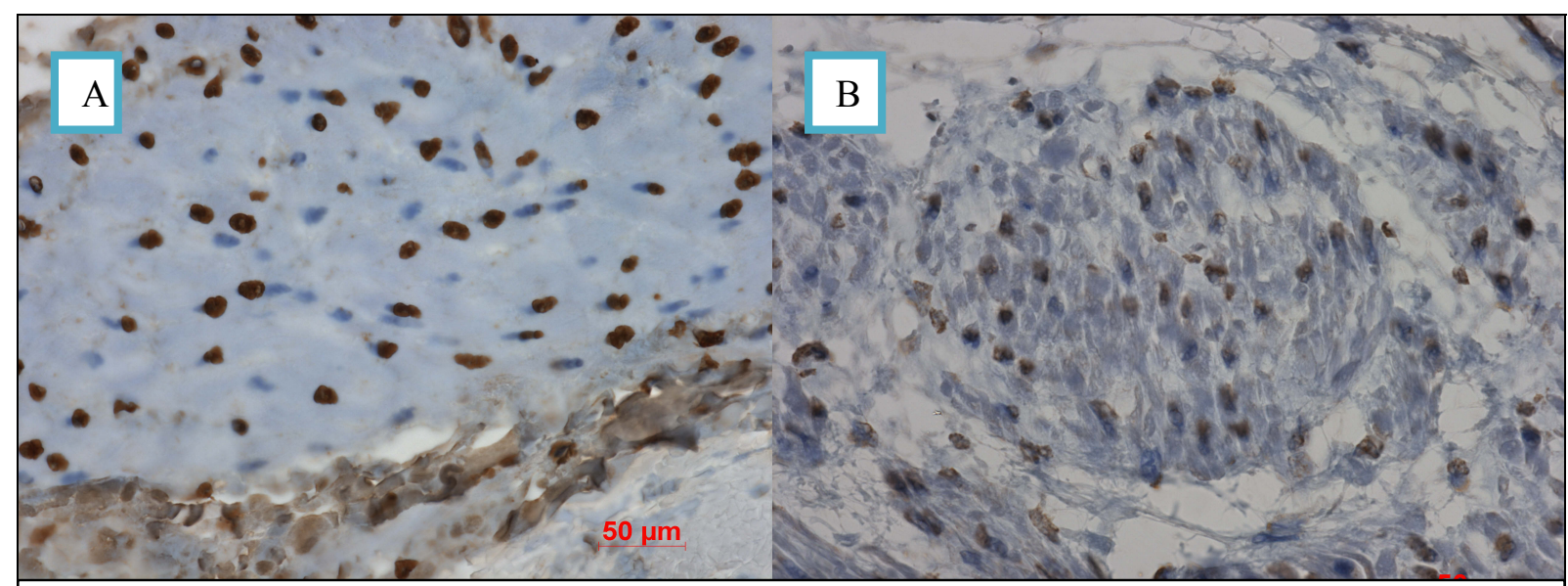

Fig. 1 A:Control case: anti-miofibroblast staining. x400.; B: Control case: anti-miofibroblast staining. $\mathrm{x} 400$

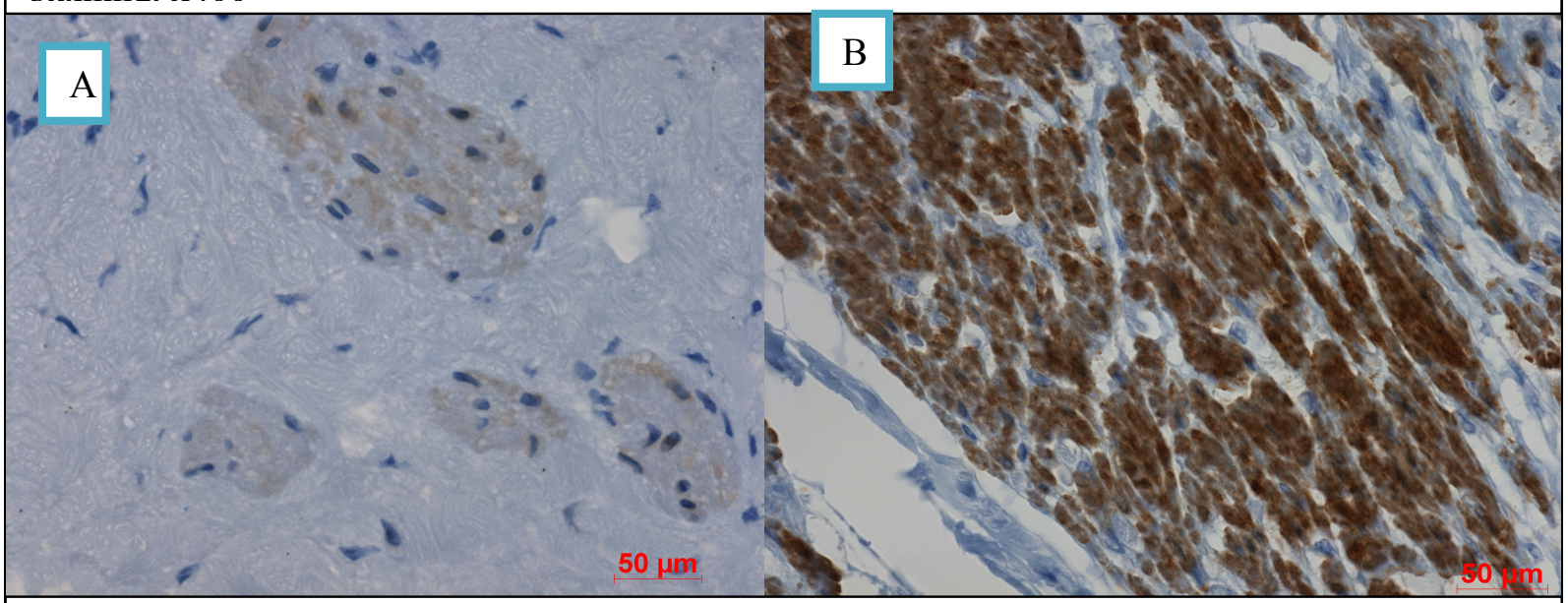

Fig. 2 A: Study case: anti-smoothelin staining. x400; B: Control case: anti-smoothelin staining. $\mathrm{x} 400$.

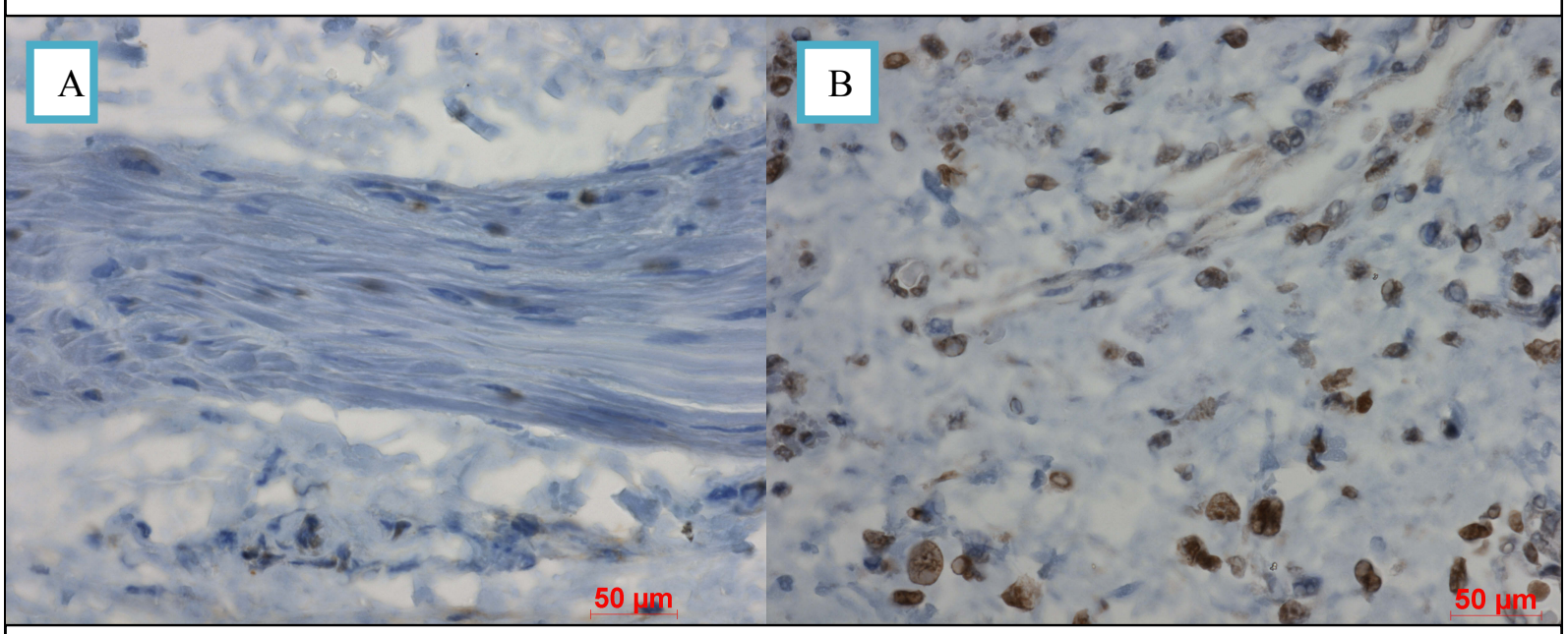

Fig. 3 A: Study case: anti- FGF2 staining. x400; B: Control case: anti- FGF 2 staining. x400; 